\title{
Energy Hypometabolism in Posterior Cingulate Cortex of Alzheimer's Patients: Superficial Laminar Cytochrome Oxidase Associated with Disease Duration
}

\author{
J. Valla, ${ }^{1,2}$ Jason D. Berndt, ${ }^{1}$ and F. Gonzalez-Lima1 \\ 1/nstitute for Neuroscience and Department of Psychology, University of Texas at Austin, Austin, Texas 78712, and \\ 2Harrington Alzheimer's Disease Research Laboratory, Arizona Alzheimer's Research Center, Phoenix, Arizona 85006
}

\begin{abstract}
Among brain regions affected in Alzheimer's disease (AD), the posterior cingulate shows the earliest and largest decrement in energy metabolism. Positron emission tomography (PET) studies have shown that these decrements appear before the onset of memory deficits or other symptoms in persons at genetic risk for AD. This study compares in vivo imaging results and in situ postmortem analyses by examining the posterior cingulate (area 23) in $15 \mathrm{AD}$ patients and 13 age-matched nondemented controls using quantitative cytochrome oxidase histochemistry as an intracellular measure of oxidative energy metabolic capacity. Each of the six layers of the posterior cingulate demonstrated a decline in cytochrome oxidase activity in AD relative to controls, whereas adjacent motor cortex showed no significant differences. This decrement did not appear to be mainly secondary to nonspecific decrement in mitochondrial enzymes, oxidative stress, cell loss, or histopathology. The cytochrome
\end{abstract}

oxidase decrement was most severe in the superficial layer I $(-39 \%)$, which demonstrated a correlation to disease duration. Covariance analyses suggest that superficial laminas undergo a functional uncoupling from the deeper layers of posterior cingulate cortex in AD, whereas no such effects are found in motor cortex or controls. These findings expand on previous results from PET studies by illuminating the layer-specific cytochrome oxidase contributions to energy hypometabolism. The findings suggest a decrement of cytochrome oxidase in posterior cingulate cortex, with progressive reduction within the superficial laminas linked to disease duration. Such decrement could contribute to some of the behavioral symptoms displayed by AD patients. This decrement appeared greater in women.

Key words: cytochrome oxidase; energy metabolism; posterior cingulate cortex; Alzheimer's disease; brain mapping; gender
Decrements in energy metabolism are one of the earliest detectable abnormalities in Alzheimer's disease (AD). Positron emission tomography (PET) studies on subjects homozygotic for the $\epsilon 4$ allele of the apolipoprotein E (APOE) gene, with no symptoms of $\mathrm{AD}$, demonstrate metabolic reductions in posterior cingulate, parietal, temporal, and prefrontal cortices (Reiman et al., 1996; Small et al., 2000). Their largest decrement was in the posterior cingulate cortex (PCC) (Reiman et al., 1996), as in the case of AD (Minoshima et al., 1994). Another PET study (Minoshima et al., 1997) confirmed that although normal subjects show intense PCC metabolic activity, early-stage AD patients suffered a PCC decrement (21-22\%) that was significantly greater than that seen in other cortical regions. This greater hypometabolism determined by PET is not merely an artifact of generalized atrophy (Ibanez et al., 1998) or AD histopathology that is lower in PCC than the other regions (Braak and Braak, 1998), although

\footnotetext{
Received Oct. 30, 2000; revised April 17, 2001; accepted April 19, 2001.

This work was supported by Texas Advanced Technology Program Grant 361 and National Institutes of Health Grant R01 NS37755 to F.G.L. We thank the Sun Health Research Institute (Sun City, AZ) and the Harvard Brain Tissue Resource Center, supported in part by Public Health Service Grant MH/NS 31862 (Belmont, MA) for donating the tissue. We also thank Alison M. Crane at University of Texas at Austin and Lucia Sue at the Sun Health Research Institute for their technical assistance. This study was submitted in partial fulfillment of the requirements for the PhD degree of J. Valla at the University of Texas at Austin.

Correspondence should be addressed to F. Gonzalez-Lima, Professor and Head, Behavioral Neuroscience, University of Texas at Austin, Mezes Hall 330, Austin, TX 78712. E-mail: gonzalezlima@psy.utexas.edu.

J. D. Berndt's present address: Neuroscience Program, University of Wisconsin, Madison, WI 53706.

Copyright (C) 2001 Society for Neuroscience $\quad 0270-6474 / 01 / 214923-08 \$ 15.00 / 0$
}

degeneration of PCC occurs in AD (Brun and Gustafson, 1976). Severity of AD symptoms is correlated with hypoactivity in PCC but not temporal regions, as measured with PET and magnetic resonance imaging (Ishii et al., 1997; Hirono et al., 1998; Alsop et al., 2000). PCC hypoperfusion was also shown by subjects with only questionable dementia who later converted to AD in longitudinal SPECT studies (Johnson et al., 1998; Kogure et al., 2000). Cingulectomy has been linked to neglect and amnesia (Watson et al., 1973), and PCC is critically involved in memory retrieval as shown by PET studies (Nyberg et al., 1996; Cabeza et al., 1997). Together these studies suggest that regionally selective hypometabolism in PCC may be an important early event in the progression of AD.

In $\mathrm{AD}$ certain regions and cells are more vulnerable than others are in terms of energy metabolism but not histopathology (Gonzalez-Lima et al., 1998ab). For example, inferior colliculus neurons in $\mathrm{AD}$ patients versus age-matched controls revealed a selective reduction in cytochrome oxidase (CO) activity in dendritic neuropil around the largest and most metabolically active cell bodies in the central nucleus (Gonzalez-Lima et al., 1997). $\mathrm{CO}$ is the mitochondrial enzyme responsible for the activation of oxygen for aerobic energy metabolism and provides an intracellular measure of oxidative metabolic capacity because it is critically tied to ATP production inside mitochondria (Wong-Riley, 1989). CO is primarily localized in mitochondria near excitatory synapses in dendrites of neurons (Wong-Riley et al., 1998). Given the selective $\mathrm{CO}$ decrements in dendritic neuropil of AD neurons, it was of interest to investigate whether $\mathrm{CO}$ decrements may be preferentially localized to dendritic-rich cortical layers. The 


\begin{tabular}{|c|c|c|c|c|c|c|c|}
\hline Subject & Age & Gender & $\begin{array}{l}\text { PMI } \\
(\mathrm{hr})\end{array}$ & $\begin{array}{l}\text { Brain } \\
\text { wt. (gm) }\end{array}$ & APOE & Clinical diagnosis/cause of death & Neuropathology diagnosis \\
\hline \multicolumn{8}{|c|}{ Alzheimer's cases } \\
\hline 3974 & 73 & $\mathrm{~F}$ & 5 & 1020 & & Prob AD/unknown & CERAD definite AD \\
\hline 9726 & 77 & $\mathrm{~F}$ & 3.5 & 900 & $3 / 4$ & Poss AD/intestinal obstruction, hypovolemia & CERAD definite AD \\
\hline 4353 & 77 & $\mathrm{~F}$ & 6.1 & 1060 & & Prob AD/unknown & CERAD definite AD \\
\hline 9701 & 78 & $\mathrm{~F}$ & 5 & 1270 & $3 / 3$ & Prob $\mathrm{AD} /$ end stages of $\mathrm{AD}$ & CERAD definite AD \\
\hline 9721 & 81 & M & 3 & 1150 & $3 / 4$ & Prob AD/pneumonia & CERAD definite AD \\
\hline 9810 & 82 & M & 3.25 & 1145 & $3 / 4$ & Prob $\mathrm{AD}, \mathrm{COPD} / \mathrm{CHF}$ & CERAD definite AD \\
\hline 9812 & 84 & M & 5.33 & 1050 & $4 / 4$ & Prob AD/CHF, inanition & CERAD definite AD \\
\hline 4179 & 84 & M & 4.5 & 1200 & & Prob AD/unknown & CERAD definite AD \\
\hline 9807 & 85 & M & 3.5 & 1220 & $3 / 3$ & Poss $\mathrm{AD}, \mathrm{CHF} / \mathrm{RF}$ & CERAD NP prob AD \\
\hline 9817 & 86 & $\mathrm{~F}$ & 4.5 & 780 & $3 / 3$ & Prob AD, depression/cardiac arrhythmia, ASHD & CERAD NP prob AD \\
\hline 9830 & 86 & $\mathrm{~F}$ & 3.5 & 1055 & $3 / 4$ & Prob AD, ASHD/cardiomyopathy & CERAD definite AD \\
\hline 9727 & 88 & M & 5 & 1120 & $2 / 3$ & Prob $\mathrm{AD} /$ end stages of $\mathrm{AD}$ & CERAD definite AD \\
\hline 9828 & 88 & M & 3 & 1170 & $3 / 3$ & Prob AD/CVA & CERAD definite AD \\
\hline 9806 & 91 & $\mathrm{~F}$ & 5 & 745 & $3 / 4$ & Prob $\mathrm{AD} /$ end stages of $\mathrm{AD}$ & CERAD definite AD \\
\hline 9818 & 91 & $\mathrm{~F}$ & 3.16 & 1010 & $3 / 3$ & Poss $\mathrm{AD}, \mathrm{COPD} / \mathrm{CHF}$ & CERAD NP prob AD \\
\hline \multicolumn{8}{|c|}{ Nondemented controls } \\
\hline 4143 & 71 & M & 11 & 1330 & & Control/unknown & CERAD NP poss AD \\
\hline 9540 & 74 & M & 3 & 1230 & $3 / 3$ & Control/cardiac arrest & Normal brain, small infarcts \\
\hline 9530 & 75 & M & 3 & 1560 & $3 / 3$ & Control/cardiopulmonary arrest & $\begin{array}{l}\text { Pathologic aging, CER AD plaque score } \\
\text { c, NP poss AD }\end{array}$ \\
\hline 9537 & 77 & M & 3.5 & 1455 & $3 / 3$ & Coronary heart disease, hypertension/CHF & Pathologic aging, CAA \\
\hline 9703 & 77 & $\mathrm{~F}$ & 4.25 & 1235 & $3 / 4$ & Control/cardiopulmonary arrest & Pathologic aging, CERAD NP poss AD \\
\hline 9904 & 77 & M & 3.5 & 1190 & $2 / 3$ & Head and neck cancer/RF & $\begin{array}{l}\text { Astrocytoma, CERAD plaque score a, } \\
\text { NP not AD }\end{array}$ \\
\hline 9527 & 78 & M & 3 & 1240 & $3 / 3$ & COPD, ASHD, pulmonary fibrosis/RF & $\begin{array}{l}\text { Normal brain, CERAD plaque score } 0 \text {, } \\
\text { NP not AD }\end{array}$ \\
\hline 9521 & 79 & M & 3 & 1260 & $3 / 3$ & CVA/intracerebral hemorrhage & Hemorrhagic infarction \\
\hline 9538 & 81 & $\mathrm{~F}$ & 3.75 & 1225 & $2 / 3$ & Control/pneumonia & $\begin{array}{l}\text { Pathologic aging, CAA, asymptomatic } \\
\text { Lewy bodies }\end{array}$ \\
\hline 9833 & 82 & $\mathrm{~F}$ & 3 & 1185 & $3 / 3$ & Lung cancer & $\begin{array}{l}\text { Subacute infarctions, CERAD plaque } \\
\text { score a, NP not AD }\end{array}$ \\
\hline 9751 & 84 & M & 4 & 1390 & $3 / 3$ & Control/cardiopulmonary arrest & CERAD NP poss AD \\
\hline 9509 & 92 & $\mathrm{~F}$ & 2 & 925 & $3 / 3$ & Normal pressure hydrocephalus with shunt & $\begin{array}{l}\text { Normal pressure hydrocephalus with } \\
\text { shunt }\end{array}$ \\
\hline 9536 & 92 & $\mathrm{~F}$ & 2 & 985 & $3 / 3$ & Degenerative joint disease/CHF & $\begin{array}{l}\text { Pathologic aging, CER AD plaque score } \\
\text { b, NP poss AD }\end{array}$ \\
\hline 4338 & 95 & $\mathrm{~F}$ & 7.05 & 1350 & & Control/unknown & Not available \\
\hline
\end{tabular}

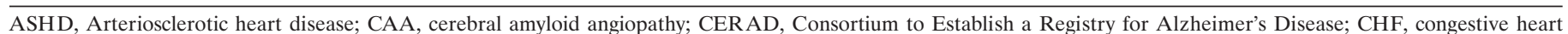
failure; COPD, chronic obstructive pulmonary disease; CVA, cerebral vascular accident; NP, CERAD Neuropathology protocol; RF, respiratory failure.

ability to do high-resolution laminar and cellular analyses is one large advantage histochemistry has compared with in vivo imaging, and as such, these techniques can complement one another. $\mathrm{CO}$ has been used to examine densitometrically the hippocampus of AD patients (Simonian and Hyman, 1993).

We assessed PCC laminar CO activity in AD patients and age-matched nondemented controls. The objectives were: (1) to confirm that $\mathrm{CO}$ activity shows more vulnerability in PCC than adjacent motor cortex, as suggested by the predominant in vivo hypometabolism of PCC in AD; (2) to investigate whether preferential $\mathrm{CO}$ decrement in $\mathrm{AD}$ may be localized to superficial cortical layers, as suggested by the predominance of $\mathrm{CO}$ in dendritic neuropil; and (3) to determine whether $\mathrm{CO}$ effects are correlated with disease duration, as expected of events relevant to AD progression.

\section{MATERIALS AND METHODS}

Subjects and samples. Frozen tissue samples from 28 subjects were either dissected by us without intermediate thawing at the Sun Health Research Institute (Sun City, AZ) (23 cases) or received frozen from the Harvard Brain Tissue Resource Center (Belmont, MA) (5 cases). The acceptable postmortem interval (PMI) for inclusion was restricted to $<12 \mathrm{hr}$ to reduce the variability of the enzyme activities. Age was restricted to $\geq 70$ years in an effort to focus on late-onset, sporadic forms of AD. Table 1 summarizes the age, gender, PMI, brain weight, APOE status, clinical diagnosis, cause of death, and neuropathology findings available for each case.

In the majority of cases, both area samples were dissected from the same section, corresponding to the medial aspect of a coronal section cut at the plane of the paracentral lobule dorsally and the posterior end of the splenium ventrally. The dorsal sample corresponded to Brodmann's area 4 in the paracentral lobule [primary motor cortex (PMC)], and the ventral sample corresponded to Brodmann's area 23 in the cingulate gyrus (PCC) (Fig. 1A). The investigated PCC is area 23 at the level of the 


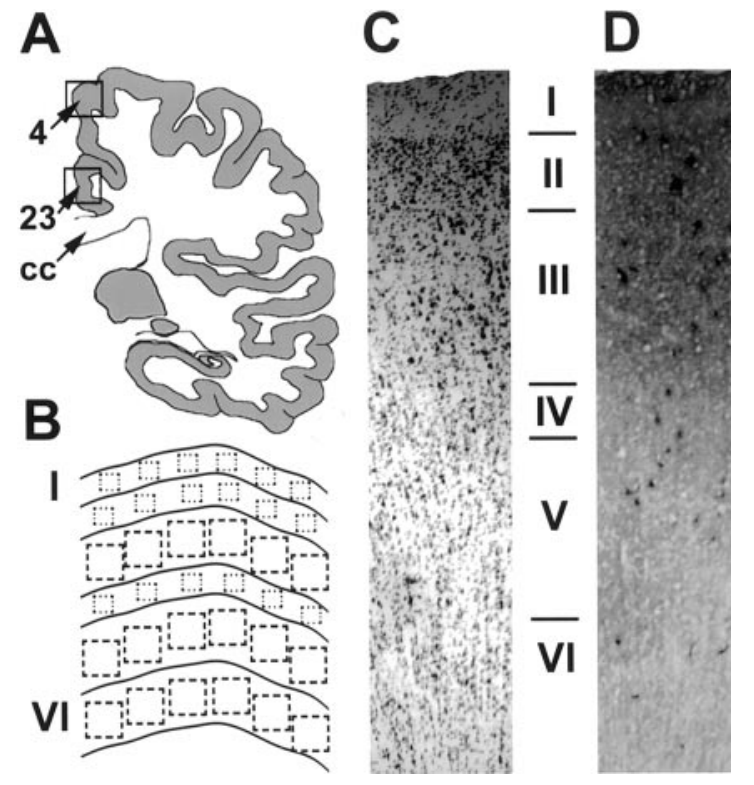

Figure 1. A, Schematic diagram indicating the sampling location for Brodmann's area 4 (PMC) and area 23 (PCC). CC, Corpus callosum. B, Schematic representation of the sampling from each layer of the cortex. $C$, Sample of Nissl-stained cortex (area 4). D, Sample of CO-stained cortex taken from a section immediately adjacent to the tissue in $C$.

splenium, not the retrosplenial cortex located posterior to the splenium. Occasionally a single area was available in a subset of the subjects, as reflected in the sample sizes reported below. This primary motor cortex region adjacent to PCC was chosen as a comparison region because it has high baseline metabolic activity similar to PCC, but it shows no significant hypometabolism in AD (Minoshima et al., 1997).

Tissue processing. Initially, several frozen sections were cut in various planes and Nissl-stained to determine a sectioning plane perpendicular to the horizontal cortical layers. Subsequently, the rest of the sample was sectioned into $40-\mu \mathrm{m}$-thick sections in this perpendicular plane and picked up on clean slides in a Frigocut 2800 cryostat at $-15^{\circ} \mathrm{C}$. Several adjacent series were created for analysis. One series was Nissl-stained with cresyl violet to aid with the determination of the laminar structure, and adjacent series were used for the histochemical stains. Experimenters were blind to the subjects' disease condition in all analyses below.

Cytochrome oxidase histochemistry. A series of sections was processed for $\mathrm{CO}$ quantitative histochemistry using previously described procedures (Gonzalez-Lima and Jones, 1994; Gonzalez-Lima and Cada, 1994, 1998; Gonzalez-Lima et al., 1997). Fresh-frozen tissue was used, and preincubation fixation was restricted to $5 \mathrm{~min}$ because of its detrimental effects on CO activity (Chalmers and Edgerton, 1989; Gonzalez-Lima and Cada, 1998). Briefly, slides were first treated in $10 \%$ sucrose phosphate buffer $(0.1 \mathrm{M}, \mathrm{pH} 7.6)$ containing $0.5 \%$ glutaraldehyde (Grade II) for $5 \mathrm{~min}$. Three changes at $5 \mathrm{~min}$ each of $10 \%$ phosphate buffer were followed by a preincubation for $10 \mathrm{~min}$ in Tris buffer $(0.05 \mathrm{M}, \mathrm{pH} 7.6)$ containing $275 \mathrm{mg} / \mathrm{l}$ cobalt chloride, $10 \%$ sucrose, and $0.5 \%$ dimethylsulfoxide. The slides were then rinsed for $5 \mathrm{~min}$ in phosphate buffer and incubated at $37^{\circ} \mathrm{C}$ for $90 \mathrm{~min}$ in $700 \mathrm{ml}$ of an oxygen-saturated reaction solution containing $350 \mathrm{mg}$ of diaminobenzidine tetrahydrochloride, 52.5 $\mathrm{mg}$ of cytochrome c, $35 \mathrm{gm}$ of sucrose, $14 \mathrm{mg}$ of catalase, and $1.75 \mathrm{ml}$ of dimethylsulfoxide in phosphate buffer. To stop the reaction and fix the tissue, a $30 \mathrm{~min}$ immersion in $10 \%$ sucrose phosphate buffer with $4 \%$ formalin ( $\mathrm{v} / \mathrm{v}$ ) was used before dehydrating, clearing (xylene), and coverslipping with Permount. Sections from AD and control subjects from the PCC were stained in a single batch, and sections from PMC in AD and controls were stained in another to remove the possibility of interbatch variability as a confound in the comparison between subject groups. In addition, there were no interbatch differences across the two regions, as verified by the optical density (OD) of $\mathrm{CO}$ activity standards included in each batch. These same standards were used to convert tissue optical density measures to $\mathrm{CO}$ activity units via a regression equation based on their optical density and spectrophotometrically determined activity (Cada et al., 1995; Gonzalez-Lima and Cada, 1998).
Figure $1 B$ shows the schematic sampling procedure for image analysis. First, the slides were coded so that the operator was blind to subjects' identity and group membership. Second, to rule out sampling bias because of better staining in the $\mathrm{CO}$-stained regions, the areas for measurement were selected in the adjacent Nissl-stained sections. Selection was based on two morphological criteria: (1) the region must be free from histological artifacts, and (2) the region must show six well defined layers. Therefore, we sampled the most histologically intact area within each sample, which may reduce the differences between groups if the differences were caused by degenerative changes. The same region selected for cell counting in the Nissl-stained section was scanned in the adjacent CO-stained section by overlapping a template with the morphological delineations and landmarks.

Laminar delineation was accomplished with a low magnification camera lucida system using a six-layer definition of the cell populations in PCC and PMC. The laminas were defined by cell typing and population density by a single rater using the cresyl violet-stained sections. Examples of Nissl- and CO-stained cortex are shown in Figure 1,C and D, respectively.

The optical density of the CO-stained sections was analyzed using an image processing system consisting of a CCD camera, DC-powered light box, image capture board (Targa), and JAVA (Jandel Scientific, Corte Madera, CA) image analysis software. The system was calibrated using an optical density step tablet (Eastman Kodak, Rochester, NY), and the images were corrected for optical distortions using a subtraction of the background illumination. Six tissue optical density readings were taken from each layer from a single section for each subject with the aid of the overlaid camera lucida sketches. Each reading consisted of the average of the pixel values in a square sampling window of variable size. The size of the window was adjusted to encompass the width of the laminas and ranged in size from $5 \times 5$ pixels $(\sim 0.12 \times 0.12 \mathrm{~mm})$ to $19 \times 19$ pixels $(\sim 0.36 \times 0.36 \mathrm{~mm})$. The measurements were taken approximately equidistant across the visible sample, and any tissue artifacts were avoided (Fig. 1B). Each subject received seven final scores: a mean of the readings from each of the six laminas and a grand mean averaging across all six laminas. Statistical values are reported as mean \pm SEM.

Comparisons between the genders and agonal states used the grand mean scores of $\mathrm{CO}$ activity. Between-group multiple comparisons of the $\mathrm{CO}$ activity in PCC and PMC were analyzed with two-tailed $t$ tests corrected for multiple comparisons with Hochberg's (1988) sharper Bonferroni procedure, as was the comparison between APOE $\epsilon 4$ carriers and noncarriers. Within-group comparisons of laminar activity within each region and group were done with the two-tailed Mann-Whitney $U$ test. All correlations presented are Pearson product moment correlations and their associated probability of being different from zero. To assess interlaminar correlations and differences between correlations, each subject's laminar data were normalized to an arbitrary overall average value of 100 to avoid artificial inflation of the correlations caused by subject variation. The correlation values found to be different from zero at $p<$ 0.05 were then compared for group differences using an r-to-z transformation corrected for multiple comparisons with Hochberg's (1988) sharper Bonferroni procedure.

Succinate dehydrogenase histochemistry. Succinate dehydrogenase (SDH), complex II of the electron transport chain, may also be affected in $\mathrm{AD}$ if our $\mathrm{CO}$ findings are attributable to a nonspecific effect on mitochondrial enzymes. To further characterize the specificity of our findings we chose to analyze the activity of this mitochondrial enzyme in the region with the largest reduction of $\mathrm{CO}$ activity, the PCC.

SDH histochemistry was performed on $40 \mu \mathrm{m}$ frozen sections adjacent to those used for $\mathrm{CO}$ histochemistry. The procedure was as follows: (1) slides were placed directly from $-20^{\circ} \mathrm{C}$ into $100 \mathrm{~mm}$ PBS with $10 \%(\mathrm{w} / \mathrm{v})$ sucrose. Three baths of $5 \mathrm{~min}$ each with graded temperature from $\sim 4-22^{\circ} \mathrm{C}$ were used to bring the slides to room temperature. (2) Slides were then transferred to PBS alone for $5 \mathrm{~min}$. (3) Slides were incubated for $8 \mathrm{~min}$ at $37^{\circ} \mathrm{C}$ in the dark in the following reaction solution: $48 \mathrm{~mm}$ sodium succinate, $1.059 \mathrm{gm}$ of EDTA (disodium salt), $0.046 \mathrm{gm}$ of sodium azide, $0.859 \mathrm{gm}$ of nitroblue tetrazolium (NBT), and $0.236 \mathrm{gm}$ of 1-methoxyphenazine methylsulfate (mPMS) in $700 \mathrm{ml}$ of double-distilled water. (4) The reaction was stopped with $10 \%$ formalin in PBS for $30 \mathrm{~min}$ at $22^{\circ} \mathrm{C}$. (5) Slides were dehydrated in graded ethanol concentrations, 30 , $50,70,95,95,100$, and $100 \%$ in double-distilled water for 5 min each. (6) Sections were cleared in xylene, with three changes, 5 min each, and coverslipped with Permount.

Through the action of mPMS, an exogenous electron carrier, SDH catalyzes the dielectronic reduction of NBT to an insoluble product, 
formazan, which can be visualized as a dark blue stain. Within a certain time course of the reaction, the accumulation of formazan does not affect the forward rate of reaction significantly. Therefore, endpoint measurements can be assumed to be linear with respect to reaction time up to 10 min, as we have determined empirically for our experimental conditions (data not shown).

The stained sections were analyzed for optical density with the same imaging system used for the $\mathrm{CO}$ analysis. Areas of measurement proximal to those used in the $\mathrm{CO}$ analysis were used. Deviations from the procedure above include that a laminar breakdown was not done; instead macroscopic average intensity measures encompassing $\sim 2 \mathrm{~mm}^{2}$ across all layers were made. The $\mathrm{CO}$ analysis was repeated in this manner to verify that no differences resulting from experimental method would skew these results, and mean CO results were the same with both methods. Data presented are mean $\pm \mathrm{SE}$, and means were tested for a significant difference using a two-tailed $t$ test.

Heme oxygenase-1 immunohistochemistry. Heme oxygenase-1 (HO1) is a putative antioxidant enzyme induced by oxidative stress (Premkumar et al., 1995). HO1 catalyzes the breakdown of heme motifs to form among other products, biliverdin, which is reduced to bilirubin, a molecule of known antioxidant properties. To determine whether our $\mathrm{CO}$ findings are attributable to a nonspecific effect of oxidative stress, HO1 immunohistochemistry was performed in the posterior cingulate cortex.

Frozen tissue sections from $\mathrm{AD}$ and control subjects were stained for the presence of HO1-reactive cells using on-the-slide immunohistochemistry. A polyclonal antibody, rabbit anti-rat $\mathrm{HO} 1\left(1: 4000 ; 16 \mathrm{hr}\right.$ at $4^{\circ} \mathrm{C}$; StressGen Biotech.Corp., Victoria, British Columbia, Canada) followed by biotinylated goat anti-rabbit $\operatorname{IgG}(1: 200)$ and avidin-biotin-HRP (Vector Elite ABC kit; Vector Laboratories, Burlingame, CA) served to label the protein. Sections were then incubated in a chromagen solution, $3,3^{\prime}$-diaminobenzidine tetrahydrochloride $(0.05 \%)$ in the presence of $\mathrm{H}_{2} \mathrm{O}_{2}(0.0015 \%)$, for $8 \mathrm{~min}$ at $22^{\circ} \mathrm{C}$. Sections were dehydrated in graded ethanol concentrations, cleared in xylenes, and coverslipped.

Using an analog camera attached to an Olympus Optical (Tokyo, Japan) BX40 bright-field microscope at $40 \times$ magnification, images were captured and imported into JAVA software for analysis. An area of measurement across all layers $\left(\sim 2 \mathrm{~mm}^{2}\right)$ proximal to that used for $\mathrm{CO}$ and SDH histochemistry was delineated using the software. A pixel-bypixel frequency histogram of ODs was generated, and a background threshold was created for each subject by selecting only those OD $>1$ SD above the mean. The object-counting feature of the software was then used to count the number of stained objects (cells) in this selected range. Adjacent stained pixels were deemed one object, and single pixel objects were eliminated from the overall count. The actual area sampled was recorded, and counts were normalized to cells per square millimeter. There were no significant group differences in the area sampled (data not shown). Data presented are mean $\pm \mathrm{SE}$, and means were tested for a significant difference using a two-tailed $t$ test.

This protocol assumes that HO1 is not reorganized from a diff use to punctate spatial distribution between the control and pathological cases. This assumption is supported by the work of other groups, which indicate that HO1 immunoreactivity is coincident with reactive astrocytosis and colocalizes with glial fibrillary acidic protein, suggesting that it might be present in glial cells responding to neurological insult (Smith et al., 1994). If this were true in the AD cases, it would result in a greater number of immunoreactive objects identified in the above protocol. Admittedly, $\mathrm{HO} 1$ is an indirect measure of oxidative insult, but this analysis does provide us with some indication that our findings are not those of generalized transcriptional downregulation.

Cell counting in cresyl violet-stained sections. To determine whether the $\mathrm{CO}$ activity decreases simply reflect cellular degeneration, counts of Nissl-stained cells were obtained from the same sections and regions used to demarcate the laminas for $\mathrm{CO}$ analysis as described in the section of the $\mathrm{CO}$ methods. Areas of measurement across all layers $\left(\sim 2 \mathrm{~mm}^{2}\right)$ used for the $\mathrm{CO}, \mathrm{SDH}$, and $\mathrm{HO} 1$ analyses were used and counted using the microscope setup and counting protocol as in the HO1 analysis. Counts were again normalized to cells per square millimeter. As in that analysis, there were no significant group differences in area sampled (data not shown). This form of counting, although not suited for indicating the actual number of cells, is sufficient to rule out group differences resulting from pathological degeneration in the sampled area (Arendt et al., 1983). Data presented are mean $\pm \mathrm{SE}$, and means were tested for a significant difference using a two-tailed $t$ test.

Amyloid plaque and neurofibrillary tangle histopathology. To investigate whether $\mathrm{CO}$ activity was related to AD histopathology a final series was stained with thioflavin S for a survey of amyloid plaque and neurofibrillary tangle pathology in the PCC. Slide-mounted sections were fixed in $4 \%$ formalin (v/v) for $30 \mathrm{~min}$, followed by pretreatment in $50 \%$ chloroform $-50 \%$ ethanol for $1 \mathrm{hr}$. The slides were passed through graded alcohols $(10 \mathrm{sec}$ each) to distilled water $(5 \mathrm{~min})$, and then to $0.1 \%$ thioflavin S (Sigma, St. Louis, MO) in distilled water (10 min). Slides were differentiated in $80 \%$ ethanol (10-12 sec), quickly rinsed in distilled water, and coverslipped with an aqueous medium. Pathology was visualized on a Zeiss Universal fluorescent microscope using a $10 \times$ objective. Severity was semiquantitatively assessed on a seven-point scale ranging from 0 (none) to 6 (frequent), using the CERAD criteria as a guideline (Mirra et al., 1991). Cored/neuritic plaques, diff use plaques, and neurofibrillary tangles were assessed separately, with each subject receiving one score for each. Scores were correlated on a subject-by-subject basis with laminar and mean $\mathrm{CO}$ activity in the AD patients using Pearson product moment correlations, and their probability of being different from zero was calculated.

\section{RESULTS}

\section{Subjects and samples}

The subject information (age, PMI, brain weight) was compared between groups using two-tailed $t$ tests. In the analysis of the PCC, the nondemented control group $(n=13)$ consisted of seven males and six females with a mean \pm SEM age of $81.2 \pm 2.1$ years, a mean PMI of $4.1 \pm 0.7 \mathrm{hr}$, and a mean brain weight of $1255 \pm$ $48 \mathrm{gm}$. The AD group $(n=15)$ consisted of seven males and eight females with a mean age of $83.4 \pm 1.4$ years, a mean PMI of $4.2 \pm$ $1.0 \mathrm{hr}$, and a mean brain weight of $1060 \pm 40 \mathrm{gm}$. AD patients' reports included confirmation of AD histopathology and, for 12 of the 15 subjects, years since diagnosis of AD (6.25 \pm 0.9 years).

In the analysis of the PMC, the nondemented control group $(n=12)$ consisted of seven males and five females with a mean age of $80.7 \pm 1.7$ years, a mean PMI of $3.2 \pm 0.2 \mathrm{hr}$, and a mean brain weight of $1240 \pm 51 \mathrm{gm}$. The AD group $(n=12)$ consisted of six males and six females with a mean age of $84.8 \pm 1.3$ years, a mean PMI of $4.0 \pm 0.8 \mathrm{hr}$, and a mean brain weight of $1051 \pm$ 48 gm. Years since diagnosis with $\mathrm{AD}=6.25 \pm 0.9$ years (identical subset of subjects as in PCC).

The AD subjects across both areas had decreased brain weight relative to their age-matched controls (Student's two-tailed $t=$ 2.7 in PMC, 3.2 in PCC; $p<0.05$ ). No significant differences existed in age across the groups in either area. No difference was found in the PMI within the PCC subjects. In PMC, the AD patients demonstrated a PMI significantly longer than controls $(t=-2.5 ; p<0.05)$; however, the actual time difference between the group means was inconsequential $(<1 \mathrm{hr})$.

APOE status was available for the majority of the subjects. It may be of interest to point out that the only control subject to carry an $\epsilon 4$ allele also had the lowest mean $\mathrm{CO}$ value $(57 \mathrm{U})$; however, in the AD patients, there were no significant differences in $\mathrm{CO}$ activity between $\epsilon 4$ carriers and noncarriers in any of the six layers, even before correction for multiple comparisons $(n=$ 12; Student's two-tailed $t$ test; $p>0.19$ ).

The mean $\mathrm{CO}$ value was used to assess the contribution of agonal disease state in the control group. Agonal state (as assessed via cause of death when available) had no apparent effect on measured $\mathrm{CO}$ activity in the controls. Those who died from relatively acute conditions (e.g., cardiac arrest, stroke) showed similar activity $(n=5 ; 78 \pm 11 \mathrm{U})$ to those with more chronic agonal conditions (e.g., cancer, chronic obstructive pulmonary disease, degenerative joint disease; $n=5 ; 104 \pm 12 \mathrm{U} ; p>0.13$; two-tailed $t$ test).

The mean $\mathrm{CO}$ value was used to assess the effect of gender within the groups. Female AD patients $(n=8)$ demonstrated significantly decreased $\mathrm{CO}$ activity relative to male $\mathrm{AD}$ patients 


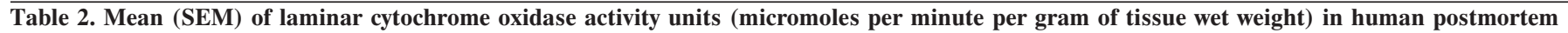
posterior cingulate (area 23) and primary motor cortex (area 4) in AD patients and age-matched controls

\begin{tabular}{|c|c|c|c|c|c|c|}
\hline & \multicolumn{3}{|c|}{ Posterior cingulate cortex } & \multicolumn{3}{|c|}{ Primary motor cortex } \\
\hline & Control & $\mathrm{AD}$ & $\%$ Difference & Control & $\mathrm{AD}$ & $\%$ Difference \\
\hline Layer I & $85(8)$ & $52(3)^{* *}$ & $-39 \%$ & $86(7)$ & $68(4)$ & $-21 \%$ \\
\hline Layer II & $88(7)$ & $60(3)^{* *}$ & $-32 \%$ & $103(7)$ & $84(4)$ & $-18 \%$ \\
\hline Layer III & $95(9)$ & $71(4)^{*}$ & $-25 \%$ & $115(6)$ & $100(4)$ & $-13 \%$ \\
\hline Layer IV & $87(8)$ & $66(3)^{*}$ & $-24 \%$ & $102(5)$ & $91(6)$ & $-11 \%$ \\
\hline Layer V & $83(7)$ & $63(3)^{*}$ & $-24 \%$ & $95(6)$ & $86(5)$ & $-9 \%$ \\
\hline Layer VI & $73(6)$ & $55(3)^{*}$ & $-25 \%$ & $86(6)$ & $76(5)$ & $-12 \%$ \\
\hline Mean & $85(7)$ & $61(2)^{* *}$ & $-28 \%$ & $98(6)$ & $84(4)$ & $-14 \%$ \\
\hline
\end{tabular}

Significantly lower than control $\left({ }^{*} p<0.05 ; * * p<0.01\right.$; two-tailed Bonferroni-corrected).

$(n=7)$ with $55 \pm 3$ and $68 \pm 2 \mathrm{U}$, respectively, in the PCC (Student's two-tailed $t$ test, $-3.3 ; p<0.01 ;-19 \%$ ). There was no similar decrement in the PMC or in the control groups in either area. We also performed a multivariate analysis to conclude that "gender" was an independent and significant factor. We had no AD severity data, but we performed this analysis using ANCOVA to covary out brain weight within the AD group and compare across gender using the layer $\mathrm{CO}$ means, after excluding one outlier identified in the regression with a Studentized residual of -3.45 . This analysis resulted in a highly significant effect of gender in the ANCOVA ( $p=0.007 ; n=7$ per group).

\section{Cytochrome oxidase laminar analysis}

The results of the laminar analysis are presented in Table 2. Light microscopic examination of the CO histochemistry in the PCC and PMC showed that our procedure stains very few cell bodies and that the stain was essentially found exclusively in neuropil distributed across all the cortical laminas. Because $\mathrm{CO}$ is a marker of excitatory synaptic activity, the predominant neuropil staining is in line with the majority of the excitatory synapses in these cortical areas being axodendritic as opposed to axosomatic (Wong-Riley et al., 1998).

In the PCC, each layer (I-VI) showed significant $\mathrm{CO}$ activity decrements in the AD patients relative to the controls $(p<0.05)$. Layers I and II showed relatively more hypometabolism $(p<$ 0.01 ), and layer I demonstrated a statistically greater decrement relative to layers III, IV, V, and VI in the AD group (Mann-
Whitney $U$ tests; $p<0.05)$. Averaging across the laminas for each subject revealed a significant overall mean activity decrease in the AD PCC of $28 \%(p<0.01)$ relative to the age-matched controls. A similar but nonsignificant trend was seen in the PMC. None of the PMC layers were significantly different between $\mathrm{AD}$ and controls.

Correlation matrices were constructed for normalized $\mathrm{CO}$ activity in each group and area (Table 3). Correlations between layers were found different from zero at $p<0.05$ in both areas and both groups. Comparing the groups using an r-to-z transformation highlighted three significant $(p<0.05$, corrected for multiple comparisons) changes in the correlative pattern in the PCC, indicating that the relationships between the laminas were altered by the disease process. The patterns of significant correlations were nearly identical across groups in the PMC with no correlations significantly different between groups after correction for multiple comparisons.

To investigate the influence of the patients' age and the duration over which they had been diagnosed with AD, those measures were correlated against $\mathrm{CO}$ activity in the PCC. There was no relationship between age and $\mathrm{CO}$ activity in $\mathrm{AD}$ or controls in the limited age range we studied. However, the duration of AD was inversely related to the severity of the decline of $\mathrm{CO}$ activity in layer I of the PCC $(r=-0.68 ; p=0.01)$, i.e., the longer the disease duration, the lower the CO activity (Table 4). No significant correlations were found in PMC.

Table 3. Interlaminar correlation matrices for normalized CO data in PCC and PMC

\begin{tabular}{|c|c|c|c|c|c|c|c|c|c|c|c|c|c|}
\hline & & \multicolumn{6}{|c|}{ Posterior cingulate cortex } & \multicolumn{6}{|c|}{ Primary motor cortex } \\
\hline & & I & II & III & IV & $\mathrm{V}$ & VI & I & II & III & IV & $\mathrm{V}$ & VI \\
\hline \multirow{5}{*}{ Control } & I & 1.00 & & & & & & 1.00 & & & & & \\
\hline & II & $0.76^{*}$ & 1.00 & & & & & $0.95^{*}$ & 1.00 & & & & \\
\hline & III & $-0.72 *$ & $-0.68^{*}$ & 1.00 & & & & -0.26 & -0.19 & 1.00 & & & \\
\hline & IV & $-0.94 *$ & $-0.83^{*}$ & $0.68 *$ & 1.00 & & & $-0.89^{*}$ & $-0.85^{*}$ & 0.41 & 1.00 & & \\
\hline & $\mathrm{V}$ & $-0.65^{*}$ & -0.37 & 0.37 & 0.42 & 1.00 & & $-0.73^{*}$ & $-0.81^{*}$ & -0.29 & 0.53 & 1.00 & \\
\hline \multirow{7}{*}{$\mathrm{AD}$} & VI & -0.25 & -0.53 & -0.16 & 0.34 & -0.03 & 1.00 & $-0.84^{*}$ & $-0.82^{*}$ & -0.17 & $0.59^{*}$ & $0.72 *$ & 1.00 \\
\hline & I & 1.00 & & & & & & 1.00 & & & & & \\
\hline & II & $0.57^{*}$ & 1.00 & & & & & $0.62^{*}$ & 1.00 & & & & \\
\hline & III & -0.10 & $0.53 * * *$ & 1.00 & & & & -0.19 & 0.15 & 1.00 & & & \\
\hline & IV & $-0.59^{*}$ & $-0.70^{*}$ & 0.09 & 1.00 & & & $-0.86^{*}$ & $-0.75^{*}$ & -0.18 & 1.00 & & \\
\hline & V & $-0.73^{*}$ & $-0.91^{*}, * *$ & -0.51 & 0.51 & 1.00 & & $-0.86^{*}$ & $-0.76^{*}$ & -0.18 & $0.85^{*}$ & 1.00 & \\
\hline & VI & $-0.63^{*}$ & $-0.73^{*}$ & $-0.68^{*}$ & 0.21 & $0.86^{*}, * *$ & 1.00 & $-0.59 *$ & $-0.81^{*}$ & -0.55 & $0.77^{*}$ & $0.77^{*}$ & 1.00 \\
\hline
\end{tabular}

*Correlation different from zero at two-tailed $p<0.05$.

$* *$ Correlation significantly different from aged control (two-tailed $p<0.05$; Bonferroni-corrected). 


\begin{tabular}{|c|c|c|}
\hline \multirow[b]{2}{*}{ Laminae } & \multicolumn{2}{|l|}{ Years } \\
\hline & Age $(n=15)$ & Duration $(n=12)$ \\
\hline I & 0.13 & $-0.68^{*}$ \\
\hline II & 0.01 & -0.41 \\
\hline III & 0.00 & -0.25 \\
\hline IV & -0.06 & -0.34 \\
\hline $\mathrm{V}$ & -0.14 & -0.21 \\
\hline VI & -0.18 & -0.16 \\
\hline
\end{tabular}

$n$, Number of subjects.

$*$ Different from zero at two-tailed $p=0.01$.

\section{Succinate dehydrogenase analysis}

$\mathrm{SDH}$, the other mitochondrial electron transport enzyme measured, showed no significant differences between AD and controls in the PCC region showing the $\mathrm{CO}$ decrement. The SDH mean \pm SEM activity (OD units $\times 100)$ was $2.81 \pm 0.17$ in controls and $2.56 \pm 0.11$ in $\mathrm{AD}$ patients $(p=0.24)$. However, $\mathrm{CO}$ activity $(\mathrm{OD}$ units $\times 100)$ reanalyzed in exactly the same way as SDH activity showed a highly significant group difference $(p=0.0017)$ between controls $(1.64 \pm 0.06)$ and AD patients $(1.39 \pm 0.03)$.

\section{Heme oxygenase-1 analysis}

HO1, the other oxidoreductase enzyme measured that is induced by oxidative stress, showed no significant differences between AD and controls in the PCC region showing the $\mathrm{CO}$ decrement. The HO1 mean \pm SEM expression (immunostained cell bodies per $\left.\mathrm{mm}^{2}\right)$ was $202 \pm 11$ in controls and $187 \pm 7$ in AD patients $(p=$ $0.30)$.

\section{Cell counting analysis}

The number of Nissl-stained cells, counted to determine whether cell loss could account for the CO decrement in the sampled PCC region, showed no significant differences between $\mathrm{AD}$ and controls. The mean \pm SEM number of cells (Nissl-stained cell bodies per square millimeter) was $483 \pm 27$ in controls and $478 \pm 14$ in AD patients $(p=0.87)$. We sampled the most histologically intact areas of the PCC to specifically rule out that any $\mathrm{CO}$ decrements could be accounted for by nonspecific cell loss or artifacts in the sampled areas. Thus, the point we made is that our sampled areas differ in $\mathrm{CO}$ but do not differ in cell body counts; it is not whether overall PCC degenerative changes may exist in $\mathrm{AD}$ as compared with controls.

\section{Histopathological analysis}

To investigate the influence of the neuropathological burden on $\mathrm{CO}$ activity in the PCC, the three semiquantitative measures of thioflavin-stained cored/neuritic plaques, diffuse plaques, and neurofibrillary tangles were correlated against the laminar and mean $\mathrm{CO}$ activity measures in the AD patients. No significant correlations were found $(p>0.05)$. In spite of significantly $(p<$ $0.05)$ greater histopathology in the AD group in every measure (cored/neuritic plaques: control, $1.54 \pm 0.14, \mathrm{AD}, 3.86 \pm 0.06$; diff use plaques: control, $1.23 \pm 0.15, \mathrm{AD}, 3.79 \pm 0.14$; tangles: control, $0.15 \pm 0.04, \mathrm{AD}, 3.21 \pm 0.19$ ), there were no relationships between subject $\mathrm{CO}$ activity and histopathological measures on a subject-by-subject basis.

We also performed a principal components analysis as used by Vogt et al. (1998). Although we did not have laminar histopathol- ogy scores and thus could not define Vogt's subtypes, we performed the principal components with the CO scores. This indicated no subtypes; that is, all six layers contributed significantly to the first principal component. A distinctive finding of our study is the lack of correlation between traditional AD histopathology and $\mathrm{CO}$ activity. Thus, it is not surprising that the $\mathrm{CO}$ findings do not resemble Vogt's subtypes based on histopathology.

\section{DISCUSSION}

The results suggest that prominent reductions in energy metabolism seen in the PCC in PET studies of probable AD cases may be caused by $\mathrm{CO}$ decrements that are more severe in the superficial laminas. This study was the first to quantify $\mathrm{CO}$ activity histochemically in PCC frozen samples from confirmed dementia cases of the AD-type and age-matched nondemented controls. As anticipated, $\mathrm{CO}$ activity showed a greater decrement in PCC than PMC, in agreement with PET studies showing prominent hypometabolism in the PCC in AD patients (Minoshima et al., 1997). A reduced $\mathrm{CO}$ activity is consistent with an energy metabolic dysfunction in the PCC of AD patients. However, our control data suggest this $\mathrm{CO}$ decrement in PCC may not be mainly attributable to nonspecific factors such as loss of cells or general decrements in other oxidoreductase enzymes. For example, the other respiratory enzyme measured, $\mathrm{SDH}$, is part of the electron transport chain in the inner mitochondrial membrane (complex II), and nonspecific local effects on CO (complex IV) should have affected SDH activity in a similar way. Similarly, it appears unlikely that other nonspecific effects such as oxidative stress and oxygen utilization in the PCC may explain our $\mathrm{CO}$ results. For example, HO1 induction should have been affected because this enzyme incorporates oxygen to heme under conditions of oxidative stress, as demonstrated in other brain regions in AD patients (Smith et al., 1994). Although our data from control analyses cannot rule out all possible explanations, together with the $\mathrm{CO}$ and PET data, the data on number of Nissl-stained cells, HO1immunostained cells, and SDH histochemical activity suggest that $\mathrm{AD}$ patients exhibit a preferential $\mathrm{CO}$ decrement in the PCC. Table 1 also included six patients with premature death (heart failure, pneumonia, cerebral vascular accident) during the course of their AD, suggesting that our $\mathrm{CO}$ findings likely do not represent a simple end-stage phenomenon. Indeed, metabolic decrements in PCC have been shown to exist very early in the course of AD (Minoshima et al., 1994, 1997; Reiman et al., 1996), and longitudinal studies show PCC dysfunction as a clinical predictor for later AD (Johnson et al., 1998; Kogure et al., 2000) that is correlated with severity of symptoms (Alsop et al., 2000).

Specificity of the CO decrement is also suggested by the lack of correlation between $\mathrm{CO}$ activity and histopathological measures. No relationship was found between $\mathrm{CO}$ activity and the presence of amyloid plaques and neurofibrillary tangles in the PCC. This dissociation is important because the early hypometabolism seen in PCC in PET studies is greater than that found in other regions, although traditional AD histopathology is lower in PCC than other regions such as the temporal cortex (Braak and Braak, 1998). In AD temporal cortex, Hatanpaa et al. (1998) quantified CO subunit III mRNA in neurons in close proximity to neuritic plaques. They found no reduction in mRNA levels in the proximal neurons or their processes compared with more plaquedistant neurons. However, Hatanpaa et al. (1996) found levels of CO mRNA decreased by $26 \%$ in neurons bearing early-stage neurofibrillary tangles compared with tangle-free neurons. Unsurprisingly, tangle-bearing neurons do not produce as much $\mathrm{CO}$, 
and likely other enzymes and proteins, as tangle-free neurons. Obviously any energy hypometabolism in these neurons may be simply secondary to the abnormal intracellular tangles. However, this did not appear to be the case in the PCC where the $\mathrm{CO}$ decrement was not associated with plaques or tangles. Thus, both the PET and $\mathrm{CO}$ data suggest that histopathology alone cannot account for PCC hypometabolism. The lack of correlation between the presence of $\mathrm{AD}$ neuropathological changes and $\mathrm{CO}$ decreases in PCC is important because it questions the linkage between traditional AD histopathology and functional disruption in $\mathrm{AD}$.

Two principal mechanisms may explain decreases in $\mathrm{CO}$ activity in AD brains: one is a nonspecific, secondary mechanism caused by reduced energy demand, and the other is a specific, primary mechanism caused by $\mathrm{CO}$ catalytic impairment. The first one may be secondary to neurofibrillary tangles or any process that may downregulate $\mathrm{CO}$ activity. For example, reduced neuronal activity and synaptic loss would downregulate $\mathrm{CO}$ and other enzymes of oxidative phosphorylation. One way to evaluate the contribution of this secondary effect is to measure other enzymes of oxidative phosphorylation, as done with SDH in this study. Clearly this secondary reduction in $\mathrm{CO}$ activity is not specific to AD (Kish et al., 1999) and can be manipulated in experimental animals by denervation or functional decreases in neuronal activity (Wong-Riley, 1989). This secondary mechanism does not appear to be the only one in PCC because a neuronal downregulation should have affected SDH activity or some of the other measures besides $\mathrm{CO}$ activity.

The second mechanism that may compromise $\mathrm{CO}$ activity in certain brain regions more than others may involve a primary $\mathrm{CO}$ catalytic impairment. We speculate that this primary mechanism appears likely to contribute to our findings in PCC for several reasons (Gonzalez-Lima et al., 1998ab). First, a catalytic abnormality in CO has been found in AD brains (Parker et al., 1994b) as well as peripheral AD tissues where it is much less likely to have resulted from secondary neuronal downregulation or another nonspecific cause. This primary $\mathrm{CO}$ impairment in AD cells cannot be attributable to nonspecific neuron atrophy or loss because it has been found in non-neural cells, such as platelets (Parker et al., 1990, 1994a), fibroblasts (Curti et al., 1997), and skeletal muscle (Gonzalez-Lima et al., 1998b). A CO decrement in these various peripheral tissues indicates that a $\mathrm{CO}$ impairment may exist in addition to any local neuropathology or neuronal atrophy in the AD brain. However, denervation and neuropathology affecting synaptic function in any given region are likely to secondarily enhance any primary $\mathrm{CO}$ decrement in that region. Second, a primary $\mathrm{CO}$ impairment in $\mathrm{AD}$ would not affect all cells equally; vulnerability would hinge on the reliance of the cell on aerobic metabolism and possibly its ability to ameliorate and/or withstand oxidative stress (Shoffner and Wallace, 1994). Thus, a CO impairment in AD may affect preferentially certain organs (like brain), cell types (like pyramidal neurons), and regions (like PCC) that are more dependent on $\mathrm{CO}$ for oxidative energy metabolism (Gonzalez-Lima et al., 1998ab). For example, systemic administration in animals of sodium azide, a $\mathrm{CO}$ activity inhibitor, have been shown to produce deficits in maze learning and memory but no motor deficits (Bennett and Rose, 1992; Bennett et al., 1992a,b), as well as different degrees of CO inhibition in different brain regions (Cada et al., 1995). Preferential vulnerability to $\mathrm{CO}$ impairments in some brain regions may also be related to their differential expression of $\mathrm{CO}$ genes (Chandrasekaran et al., 1994). Therefore, perhaps the most rea- sonable explanation for the preferential hypometabolism observed in the PCC in PET studies and in the current study may be a synergy between a primary $\mathrm{CO}$ catalytic abnormality and a secondary neuronal downregulation caused by atrophy and denervation of synapses. Separating the contribution of each of these factors to the overall decrease in $\mathrm{CO}$ activity may be difficult, but effort should be dedicated to this end because $\mathrm{CO}$ could be an attractive target for therapeutic intervention.

This study also showed that $\mathrm{CO}$ decrements in AD were localized mainly to layer I. This is a synapse-rich molecular layer with predominant dendritic neuropil as opposed to cell bodies, a fact that may contribute to its vulnerability. Aging is the major risk factor for AD; so it is interesting that layer I appears most vulnerable to aging effects. Peters et al. (1998) showed a 30-60\% reduction in synapse density in layer I in old monkeys, particularly indicating that spiny synapses that belong to the apical dendritic tufts of pyramidal cells are degenerating in old age. In the PCC, pyramidal neurons most vulnerable to atrophy in AD have their tufts of apical dendrites in layer I (Brun and Englund, 1981). Pyramidal cells also show the greatest degree of neurofibrillary tangle histopathology in AD (Hof et al., 1990; Van Hoesen, 1990; Braak and Braak, 1998). A combination of layer 1 aging effects (Peters et al., 1998) and AD-vulnerable pyramidal neurons with dendrites in layer I may also contribute to the preferential $\mathrm{CO}$ decrements in layer I in AD compared with age-matched controls. The observed $\mathrm{CO}$ decrement in layer I was correlated with disease duration, as expected of events relevant to AD progression. It would also be beneficial to assess the relationship between $\mathrm{CO}$ activity and dementia severity. To our knowledge, no such work has been done to date, and no severity information was available for our subjects. However $\mathrm{CO}$ decrement in the PCC was correlated with disease duration, and disease duration is accompanied by a progressive increase in symptom severity and PCC hypoperfusion (Alsop et al., 2000), reinforcing the possibility that dementia severity could be related to $\mathrm{CO}$ decrements.

Finally, CO activity was $\sim 20 \%$ lower in the PCC of female compared with male AD subjects. This gender effect was ADspecific in that the control subjects did not demonstrate such a disparity and region-specific in that it was not found in the PMC in either group. Demographic studies support the idea that females have an increased vulnerability to AD (Launer et al., 1999; Letenneur et al., 1999). It is hoped that CO may soon become a useful marker of metabolic vulnerability in subjects at risk for AD using new in vivo noninvasive imaging (Heekeren et al., 1999).

\section{REFERENCES}

Alsop DC, Detre JA, Grossman M (2000) Assessment of cerebral blood flow in Alzheimer's disease by spin-labeled magnetic resonance imaging. Ann Neurol 47:93-100.

Arendt T, Bigl V, Arendt A, Tennstedt A (1983) Loss of neurons in the nucleus basalis of Meynert in Alzheimer's disease, paralysis agitans and Korsakoff's Disease. Acta Neuropathologica 61:101-108.

Bennett MC, Rose GM (1992) Chronic sodium azide treatment impairs learning of the Morris water maze task. Behav Neural Biol 58:72-75.

Bennett MC, Diamond DM, Parker Jr WD, Stryker SL, Rose GM (1992a) Inhibition of cytochrome oxidase impairs learning and hippocampal plasticity: a novel animal model of Alzheimer's disease. In: Alzheimer's disease therapy: a new generation of progress (Simpkins J, Crews FT, Meyer EM, eds), pp 485-501. New York: Plenum.

Bennett MC, Diamond DM, Stryker SL, Parks JK, Parker Jr WD (1992b) Cytochrome oxidase inhibition: a novel animal model of Alzheimer's disease. J Geriatr Psychiatry Neurol 5:93-101.

Braak H, Braak E (1998) Evolution of neuronal changes in the course of Alzheimer's disease. J Neural Transm [Suppl] 53:127-140.

Brun A, Englund E (1981) Regional pattern of degeneration in Alzheimer's disease: neuronal loss and histopathological grading. Histopathology 5:549-564.

Brun A, Gustafson L (1976) Distribution of cerebral degeneration in 
Alzheimer's disease. A clinico-pathological study. Arch Psychiatr Nervenkr 223:15-33.

Cabeza R, Grady CL, Nyberg L, McIntosh AR, Tulving E, Kapur S, Jennings JM, Houle S, Craik FI (1997) Age-related differences in neural activity during memory encoding and retrieval: a positron emission tomography study. J Neurosci 17:391-400.

Cada A, Gonzalez-Lima F, Rose GM, Bennett MC (1995) Regional brain effects of sodium azide treatment on cytochrome oxidase activity: a quantitative histochemical study. Metab Brain Dis 10:303-319.

Chalmers GR, Edgerton VR (1989) Marked and variable inhibition by chemical fixation of cytochrome oxidase and succinate dehydrogenase in single motoneurons. J Histochem Cytochem 37:899-901.

Chandrasekaran K, Giordano T, Brady DR, Stoll J, Martin LJ, Rapoport SI (1994) Impairment in mitochondrial cytochrome oxidase gene expression in Alzheimer disease. Brain Res Mol Brain Res 24:336-340.

Curti D, Rognoni F, Gasparini L, Cattaneo A, Paolillo M, Racchi M (1997) Oxidative metabolism in cultured fibroblasts derived from sporadic Alzheimer's disease (AD) patients. Neurosci Lett 236:13-16.

Gonzalez-Lima F, Cada A (1994) Cytochrome oxidase activity in the auditory system of the mouse: a qualitative and quantitative histochemical study. Neuroscience 63:559-578.

Gonzalez-Lima F, Cada A (1998) Quantitative histochemistry of cytochrome oxidase activity: theory, methods, and regional brain vulnerability. In: Cytochrome oxidase in neuronal metabolism and Alzheimer's disease (Gonzalez-Lima F, ed), pp 55-90. New York: Plenum.

Gonzalez-Lima F, Jones D (1994) Quantitative mapping of cytochrome oxidase activity in the central auditory system of the gerbil: a study with calibrated activity standards and metal-intensified histochemistry. Brain Res 660:34-49.

Gonzalez-Lima F, Valla J, Matos-Collazo S (1997) Quantitative cytochemistry of cytochrome oxidase and cellular morphometry of the human inferior colliculus in control and Alzheimer's patients. Brain Res 752:117-126.

Gonzalez-Lima F, Valla J, Cada A (1998a) Brain cytochrome oxidase activity and how it relates to the pathophysiology of memory and Alzheimer's disease. In: Free radicals, oxidative stress and antioxidants: pathological and physiological significance (Ozben T, ed), pp 205-227. New York: Plenum.

Gonzalez-Lima F, Valla J, Jorandby L (1998b) Cytochrome oxidase inhibition in Alzheimer's disease. In: Cytochrome oxidase in neuronal metabolism and Alzheimer's disease (Gonzalez-Lima F, ed), pp 171201. New York: Plenum.

Hatanpaa K, Brady DR, Stoll J, Rapoport SI, Chandrasekaran K (1996) Neuronal activity and early neurofibrillary tangles in Alzheimer's disease. Ann Neurol 40:411-420.

Hatanpaa K, Chandrasekaran K, Brady DR, Rapoport SI (1998) No association between Alzheimer plaques and decreased levels of cytochrome oxidase subunit mRNA, a marker of neuronal energy metabolism. Brain Res Mol Brain Res 59:13-21.

Heekeren HR, Kohl M, Obrig H, Wenzel R, von Pannwitz W, Matcher SJ, Dirnagl U, Cooper CE, Villringer A (1999) Noninvasive assessment of changes in cytochrome-c oxidase oxidation in human subjects during visual stimulation. J Cereb Blood Flow Metab 19:592-603.

Hirono N, Mori E, Ishii K, Ikejiri Y, Imamura T, Shimomura T, Hashimoto M, Yamashita H, Sasaki M (1998) Hypofunction in the posterior cingulate gyrus correlates with disorientation for time and place in Alzheimer's disease. J Neurol Neurosurg Psych 64:552-554.

Hochberg Y (1988) A sharper Bonferroni procedure for multiple tests of significance. Biometrika 75:800-802.

Hof PR, Cox K, Morrison JH (1990) Ouantitative analysis of a vulnerable subset of pyramidal neurons in Alzheimer's disease: I. Superior frontal and inferior temporal cortex. J Comp Neurol 301:44-54.

Ibanez V, Pietrini P, Alexander GE, Furey ML, Teichberg D, Rajapakse JC, Rapoport SI, Schapiro MB, Horwitz B (1998) Regional glucose metabolic abnormalities are not the result of atrophy in Alzheimer's disease. Neurology 50:1585-1593.

Ishii K, Sasaki M, Yamaji S, Sakamoto S, Kitagaki H, Mori E (1997) Demonstration of decreased posterior cingulate perfusion in mild Alzheimer's disease by means of $\mathrm{H} 215 \mathrm{O}$ positron emission tomography. Eur J Nucl Med 24:670-673.

Johnson KA, Jones K, Holman BL, Becker JA, Spiers PA, Satlin A, Albert MS (1998) Preclinical prediction of Alzheimer's disease using SPECT. Neurology 50:1563-1571.

Kish SJ, Mastrogiacomo F, Guttman M, Furukawa Y, Taanman JW, Dozic S, Pandolfo M, Lamarche J, DiStefano L, Chang LJ (1999) Decreased brain protein levels of cytochrome oxidase subunits in Alzheimer's disease and in hereditary spinocerebellar ataxia disorders: a nonspecific change? J Neurochem 72:700-707.
Kogure D, Matsuda H, Ohnishi T, Asada T, Uno M, Kunihiro T, Nakano S, Takasaki M (2000) Longitudinal evaluation of early Alzheimer's disease using brain perfusion SPECT. J Nuc Med 41:1155-1162.

Launer LJ, Andersen K, Dewey ME, Letenneur L, Ott A, Amaducci LA, Brayne C, Copeland JR, Dartigues JF, Kragh-Sorensen P, Lobo A, Martinez-Lage JM, Stijnen T, Hofman A (1999) Rates and risk factors for dementia and Alzheimer's disease: results from EURODEM pooled analyses. EURODEM Incidence Research Group and Work Groups. European Studies of Dementia. Neurology 52:78-84.

Letenneur L, Gilleron V, Commenges D, Helmer C, Orgogozo JM, Dartigues JF (1999) Are sex and educational level independent predictors of dementia and Alzheimer's disease? Incidence data from the PAQUID project. J Neurol Neurosurg Psychiatry 66:177-183.

Minoshima S, Foster NL, Kuhl DE (1994) Posterior cingulate cortex in Alzheimer's disease. Lancet 344:895.

Minoshima S, Giordani B, Berent S, Frey KA, Foster NL, Kuhl DE (1997) Metabolic reduction in the posterior cingulate cortex in very early Alzheimer's disease. Ann Neurol 42:85-94.

Mirra SS, Heyman A, McKeel D, Sumi SM, Crain BJ, Brownlee LM, Vogel FS, Hughes JP, van Belle G, Berg L (1991) The consortium to establish a registry for Alzheimer's disease (CERAD). Part II. Standardization of the neuropathologic assessment of Alzheimer's disease. Neurology 41:479-486.

Nyberg L, McIntosh AR, Cabeza R, Nilsson LG, Houle S, Habib R, Tulving E (1996) Network analysis of positron emission tomography regional cerebral blood flow data: ensemble inhibition during episodic memory retrieval. J Neurosci 16:3753-3759.

Parker Jr WD, Filley CM, Parks JK (1990) Cytochrome oxidase deficiency in Alzheimer's disease. Neurology 40:1302-1303.

Parker Jr WD, Mahr NJ, Filley CM, Parks JK, Hughes MA, Young DA, Cullum CM (1994a) Reduced platelet cytochrome c oxidase activity in Alzheimer's disease. Neurology 44:1086-1090.

Parker Jr WD, Parks J, Filley CM, Kleinschmidt-DeMasters BK (1994b) Electron transport chain defects in Alzheimer's disease brain. Neurology 44:1090-1096.

Peters A, Sethares C, Moss MB (1998) The effects of aging on layer 1 in area 46 of prefrontal cortex in the rhesus monkey. Cereb Cortex 8:671-684

Premkumar DR, Smith MA, Richey PL, Petersen RB, Castellani R, Kutty RK, Wiggert B, Perry G, Kalaria RN (1995) Induction of heme oxygenase- 1 mRNA and protein in neocortex and cerebral vessels in Alzheimer's disease. J Neurochem 65:1399-1402.

Reiman EM, Caselli RJ, Yun LS, Chen K, Bandy D, Minoshima S, Thibodeau SN, Osborne D (1996) Preclinical evidence of Alzheimer's disease in persons homozygous for the epsilon 4 allele for apolipoprotein E. N Engl J Med 334:752-758.

Shoffner JM, Wallace DC (1994) Oxidative phosphorylation diseases and mitochondrial DNA mutations: diagnosis and treatment. Annu Rev Nutr 14:535-568.

Simonian NA, Hyman BT (1993) Functional alterations in Alzheimer's disease: diminution of cytochrome oxidase in the hippocampal formation. J Neuropathol Exp Neurol 52:580-585.

Small GW, Ercoli LM, Silverman DH, Huang SC, Komo S, Bookheimer SY, Lavretsky H, Miller K, Siddarth P, Rasgon NL, Mazziotta JC, Saxena S, Wu HM, Mega MS, Cummings JL, Saunders AM, PericakVance MA, Roses AD, Barrio JR, Phelps ME (2000) Cerebral metabolic and cognitive decline in persons at genetic risk for Alzheimer's disease. Proc Natl Acad Sci USA 97:6037-6042.

Smith MA, Kutty RK, Richey PL, Yan SD, Stern D, Chader GJ, Wiggert B, Petersen RB, Perry G (1994) Heme oxygenase-1 is associated with the neurofibrillary pathology of Alzheimer's disease. Am J Pathol $145: 42-47$

Van Hoesen GW (1990) The dissection by Alzheimer's disease of cortical and limbic neural systems relevant to memory. In: Brain organization and memory: cells, systems, and circuits (McGaugh JL, Weinberger NM, and Lynch G, eds), pp 234-261. New York: Oxford UP.

Vogt BA, Vogt LJ, Vrana KE, Gioia L, Meadows RS, Challa VR, Hof PR, Van Hoesen GW (1998) Multivariate analysis of laminar patterns of neurodegeneration in posterior cingulate cortex in Alzheimer's disease. Exp Neurol 153:8-22.

Watson RT, Heilman KM, Cauthen JC, King FA (1973) Neglect after cingulectomy. Neurology 23:1003-1007.

Wong-Riley MTT (1989) Cytochrome oxidase: an endogenous metabolic marker for neuronal activity. Trends Neurosci 12:94-101.

Wong-Riley MTT, Nie F, Hevner RF, Liu S (1998) Brain cytochrome oxidase: functional significance and bigenomic regulation in the CNS. In: Cytochrome oxidase in neuronal metabolism and Alzheimer's disease (Gonzalez-Lima F, ed), pp 1-53. New York: Plenum. 